\title{
Collagen crosslink excretion and staging of oral cancer
}

\author{
ING Springer*,', H Terheyden', A Dunsche', N Czech², MAA Suhr ${ }^{3}$, M Tiemann ${ }^{4}$, J Hedderich ${ }^{5}$ and Y Açil' \\ 'Department of Oral and Maxillofacial Surgery, University of Kiel, Arnold-Hellerstr 16, D-24 05 Kiel, Germany; ${ }^{2}$ Department of Nuclear Medicine, \\ University of Kiel, Arnold-Heller Straße 9, D-24 05 Kiel, Germany; ${ }^{3}$ Department of Oral and Maxillofacial Surgery, Klinikum Nord, Tangstedter \\ Landstrasse 400, 22417 Hamburg, Germany; ${ }^{4}$ Department of Surgical Pathology, University of Kiel, Niemannsweg II, 24 I 05 Kiel, Germany; ${ }^{5}$ nstitute \\ of Medical Informatics and Statistics, University of Kiel, Brunswiker Str. 10, 24105 Kiel, Germany
}

\begin{abstract}
Lysylpyridinoline (LP) and hydroxylysylpyridinoline (HP) are collagen crosslink residues of which the urinary concentration reflects the level of connective-tissue turnover. HP is ubiquitous in tissue, whereas LP is specific for bone. The purpose of this investigation was to assess the sensitivity and specificity of an increased urinary concentration of both HP and LP in indicating infiltration of mandibular bone by an oral squamous cell carcinoma (OSCC) or recurrence of the disease after successful therapy. We investigated the history and urine levels in 116 adult patients, who were divided into the following groups. Group I: patients with OSCC with bone infiltration ( $n=17)$; group 2: patients with confirmed OSCC $(n=12)$ without evidence of bone infiltration; group 3: patients with recurrence of an OSCC $(n=13)$; group 4: patients without clinical evidence of disease $(n=74)$. The range and upper limit of normal values $\left(H P_{\max }\right.$ and $L P_{\max }$ ) were measured from the normal controls in group 4. Levels of $L P$ and HP were measured by HPLC and fluorescence detection. There was a significant difference in the average urinary levels of LP and HP between groups I-4 $(P<0.00 \mathrm{I})$. The presence of mandibular bone infiltration could be detected with a sensitivity and specificity of I00\% when comparing groups I and 2 . Presence of tumour tissue could be detected with a sensitivity of $90 \%$. In conclusion, a normal LP concentration in patients with an OSCC strongly suggests that bone invasion by the disease has not taken place. If both urinary HP and LP are elevated, disease recurrence is highly likely.

British Journal of Cancer (2003) 88, I I05- I I I 0. doi: I 0. 1038/sj.bjc.6600873 www.bjcancer.com

(c) 2003 Cancer Research UK
\end{abstract}

Keywords: pyridinoline; collagen crosslinks; staging; oral squamous cell carcinoma; bone infiltration

Turnover and resorption of collagen in tissues adjacent to malignant disease processes may be increased (Body and Delmas, 1992). Hydroxylysylpyridinoline (HP) and lysylpyridinoline (LP) are two nonreducible crosslinks of mature collagen, which are formed by a sequence of post-translational modifications. Hydroxylyslpyridindine is a derivative of three residues of hydroxylysine and is present in virtually all mature tissues (tendon, vessel wall, cartilage, dentine and bone). Lysylpyridindine is a derivative of two residues of hydroxylysine and one residue of lysine and is found principally in dentine and bone. (Body and Delmas, 1992; Eyre, 1992; Miyamoto et al, 1994; Acil et al, 1996, 2000; Papatheofanis, 1997; Tamada et al, 2001). It has been suggested that the detection of LP in the serum or urine may be a helpful marker in establishing and possibly quantifying bone matrix resorption (Body and Delmas, 1992; Miyamoto et al, 1994; Vinholes et al, 1996; Papatheofanis, 1997; Tamada et al, 2001). The measurement of HP and, in particular, LP has been shown to be valuable in detecting the presence and/or extent of bony metastases in patients with multiple myeloma, carcinoma of the breast, lung, prostate gland, kidney, throat and digestive tract (Takeuchi et al, 1996; Papatheofanis, 1997; Walne et al, 1997; Yoshida et al, 1997; Coleman et al, 1999; Marttunen et al, 1999; Tamura et al, 1999; Woitge et al, 1999, 2001; Demers et al, 2000;

*Correspondence: Dr ING Springer; E-mail: springer@mkg.uni-kiel.de Revised 20 November 2002; accepted 9 January 2003
Fontana and Delmas, 2000; Liubimova et al, 2000; Izumi et al, 2001; Tamada et al, 2001).

Treatment decisions in oral cancer are influenced by the infiltration of mandibular bone, with consequently increased operating time and extent of resection, where surgery is indicated (Reichart and Philipsen, 1999; Baker et al, 2001; Hong et al, 2001; Schimmele, 2001). Computertomography, fluorodeoxyglucose (FDG) positron emission tomography (PET) or technetium $99 \mathrm{~m}$ methylene diphosphonate (MDP) single-photon emission computed tomography (SPECT) are the methods of choice in assessing bony involvement of malignant disease (Minn et al, 1993; Huntley et al, 1996; Schimming et al, 2001). Measurement of the concentration of LP in the urine may be an alternative. Oral squamous cell carcinoma (OSCC) may result in increased concentrations of HP in the urine, whether mandibular bone has been infiltrated or not. We suggest that the measurement of HP and LP in the urine of recall patients after apparently successful treatment for oral cancer may assist in the screening for recurrence. We discuss the role of collagen crosslinks as markers of tissue turnover in their potential role in the staging of oral cancer.

\section{MATERIALS AND METHODS}

Patients were recruited from the Tumour Clinic in the Department of Oral and Maxillofacial Surgery, University of Kiel, Germany. In all, 475 urine samples from 116 patients (age range 36-91 years) 
were analysed. A prospective double-blind study was designed, and the patients divided into four groups as follows:

Group 1 ( $n=17)$ : patients with OSCC (pT4 N1-2 M0) with bone infiltration (10 males, seven females; eight patients 39-60 years of age; nine patients $61-88$ years of age).

Group $2(n=12)$ : patients with OSCC (pT1-3 N0-1) with no bone infiltration (seven males, five females, six patients 44-60 years of age; six patients 61-81 years of age).

Group $3(n=13)$ : patients with recurrence of the disease, in seven of whom (group $3 b$ ) we obtained samples up to 6 months prior to the confirmation of the recurrence (nine males, four females; four patients 52-60 years of age; nine patients 61-83 years of age).

Group $4(n=74)$ : patients without disease (53 males, 21 females; 28 patients $36-60$ years of age; 56 patients $61-91$ years of age), control group.

Patients who were seen in our follow-up programme after apparently adequate initial treatment of OSCC were used as controls if treatment had been completed more than a year prior to entry into the study. In this group, an $\mathrm{R}_{0}$-resection and $<\mathrm{T} 2$ disease was required for inclusion as a control. Patients who had a history of malignancy other than OSCC or had alterations in renal function (urea $>50 \mathrm{mg} \mathrm{dl}^{-1}$, creatinine $>1.2 \mathrm{mg} \mathrm{dl}^{-1}$ ) were excluded. Recall patients were excluded if a previous recurrence had been documented and successfully treated. Patients were also excluded if they had had a surgical procedure or a trauma less than 6 months prior to entry into the study (see results). Patients of groups $1-3$ were staged by ultrasound and computed tomography. Technetium $99 \mathrm{~m}$ MDP bone scans with planar imaging and SPECTs were additionally performed on all patients included in the study. A chest X-ray, abdominal ultrasound, endoscopic examination of the upper aerodigestive tract and gynaecological examinations in female patients completed the assessment of concomitant disease processes. The histopathological examination was performed by a single pathologist.

\section{Preparation and hydrolysis of urine}

Samples were taken in the morning and stored at $-70^{\circ} \mathrm{C}$ until further processing. All laboratory investigations were performed in a single laboratory. Hydroxylyslpyridinoline and LP levels seem to be stable in urine samples for over 10 years if samples are stored at this temperature. The urine samples were centrifuged at 1000 r.p.m. for $5 \mathrm{~min}$. A measure of $2 \mathrm{ml}$ of supernatant was lyophilized and subsequently redissolved in $2 \mathrm{ml} 6 \mathrm{~N}$ hydrochloric acid. The samples were hydrolysed at $110^{\circ} \mathrm{C}$ for $24 \mathrm{~h}$ and centrifuged at 1000 r.p.m. for $5 \mathrm{~min}$. A volume of $1 \mathrm{ml}$ of each hydrolysate was added to a mixture of $1 \mathrm{ml}$ glacial acetic acid, $2 \mathrm{ml}$ n-butan-1-ol and $5 \mathrm{ml} \mathrm{10 \%} \mathrm{CF-1-slurry} \mathrm{(fibrous} \mathrm{cellulose} \mathrm{powder,}$ Whatman, Maidstone, UK). The CF-1-slurry was composed of $10 \%$ $\left(\mathrm{wv}^{-1}\right)$ CF-1 in a mobile phase containing $n$-butan-1-ol, glacial acetic acid and water $(4: 1: 1)$. A column was prepared by adding the mixture of hydrolysate and CF-1-slurry as described above to an econo-column polyprop $(40 \times 8 \mathrm{~mm}$, Bio-Rad München, Germany). The resin was washed three times with $5 \mathrm{ml}$ of the mobile phase. Subsequently, the pyridinium-containing eluate was eluted from the column with $3 \times 2 \mathrm{ml}$ distilled water into a $15 \mathrm{ml}$ plastic tube and traces of $n$-butan-1-ol were removed from the surface of the eluate. Thereafter, the lyophylised eluate was redissolved in $1 \mathrm{ml} 0.22 \%\left(\mathrm{vv}^{-1}\right) n$-heptafluorobutyric acid (HFBA) and centrifuged at 1000 r.p.m. for $5 \mathrm{~min}$. A volume of $200 \mu \mathrm{l}$ of the sample was analysed by HPLC (as below). The variations within and between series were 2 and $4.8 \%$, respectively.

\section{Pyridinoline standards}

The HPs and LPs were quantified by external standards gained from a commercially available adult bovine bone gelatin (Deutsche
Gelatine-Fabriken Stoess, Eberbach/Baden, Germany) prior to the application of the samples to the chromatography system. Hydroxylyslpyridinoline and LP were purified by a preparative reverse-phase-column HPLC and the degree of purity was verified by amino-acid analysis ( $>98 \%$ of dry weight) according to a method previously described (Acil and Müller, 1994; Acil et al, 1996, 2000) Serial dilutions of HP between 0 and 2250 $\left(\mathrm{pmol} \mathrm{nmol}^{-1}\right)$ and LP 0 and $1200\left(\mathrm{pmol} \mathrm{ml}^{-1}\right)$ were analysed to demonstrate the linear response of the external standards.

\section{Analysis of HP and LP by reverse-phase-column HPLC}

Chromatography was performed on a Dionex HPLC system (Idstein, Germany) at $22^{\circ} \mathrm{C}$. The flow rate was $0.7 \mathrm{ml} \mathrm{min}^{-1}$ using two continually degassed solvents: (A) $0.22 \%\left(\mathrm{v} \mathrm{v}^{-1}\right) n$ - HFBA in water and (B) $0.22 \%\left(\mathrm{vv}^{-1}\right)$ HFBA in $80 \%\left(\mathrm{vv}^{-1}\right)$ acetonitrile. The resin (Inertsil ODS-3 $5 \mu \mathrm{m}, 125 \times 4.6 \mathrm{~mm} \mathrm{C}_{18}$ ) was equilibrated with $18 / 82 \%$ solvent $B$ to solvent A prior to the application of the sample $\left(200 \mu \mathrm{l}\right.$ in $0.22 \%\left(\mathrm{v} \mathrm{v}^{-1}\right)$ HFBA in water $)$. The column was washed with $18 / 82 \%\left(\mathrm{vv}^{-1}\right.$; B/A) for $5 \mathrm{~min}$ and developed with the following step gradients:

(1) $18-20 \%$ solvent B over 20 min; the peaks of HP and LP were eluted at approximately 18 and $20 \mathrm{~min}$;

(2) $20-25 \%$ solvent $\mathrm{B}$ in $4 \mathrm{~min}$;

(3) $25-100 \%$ solvent B in 1 min plus washing of the column for another 5 min with $100 \%$ solvent B;

(4) $100-18 \%$ over solvent B in $4 \mathrm{~min}$; 1 min was used for column equilibration, thereafter. The next sample was injected after $35 \mathrm{~min}$.

Fluorescence measurements were obtained with an excitation wavelength of $297 \mathrm{~nm}$ and emission wavelength of $397 \mathrm{~nm}$ and the concentrations of HP and LP expressed in $\mathrm{pmol} \mathrm{ml}^{-1}$. After dilution $(1: 20)$ of $1 \mathrm{ml}$ of patient urine, the urinary creatinine content was measured by the colorimetric Jaffé-reaction and expressed in $\mathrm{mg} \mathrm{dl}^{-1}$ (Beckman Creatinine Analyzer 2, USA). The urinary content of HP and LP was expressed in relation to the urinary creatinine concentration, that is, in $\mathrm{nmol} \mathrm{mmol}^{-1}$ creatinine.

\section{Statistical methods}

There was no statistically significant difference between the sexes, and a normal distribution of the urinary concentrations of HP and LP was seen in groups 1-4. An analysis of variance (ANOVA) was performed to evaluate statistically significant differences between groups 1-4. Multiple comparisons were performed according to Games-Howell. Since the variance of groups 1-4 appeared to be not strictly homogeneous (Figure $1 \mathrm{~A}$ and $\mathrm{B}$ ), medians were calculated and the significance of differences tested according to Kruskal-Wallis to confirm results obtained by the ANOVA. The arithmetic mean of the urinary levels of HP and LP of the control group (group 4) was calculated and 1.96 standard deviations used to define the normal range. These upper limits were called $\mathrm{HP}_{\max }$ and $\mathrm{LP}_{\text {max }}$. The urinary concentration beyond $\mathrm{HP}_{\max }$ and $\mathrm{LP}_{\max }$ was elevated with a $P<0.025$. The sensitivity and specificity in respect of the differentiation of groups 1 (OSCC with bone infiltration) and 2 (OSCC without bone infiltration) based on $\mathrm{HP}_{\max }$ and $\mathrm{LP}_{\max }$ as cutoff points were calculated. The suspicion of mandibular bone infiltration on the basis of increased values of HP or LP that could not be confirmed by diagnostic measures such as those described above (first part of the Materials and Methods section) was classified as being false positive. Similarly, increased values of HP and LP in patients without tumour recurrence were classified as being false positive. Any indication of mandibular bone infiltration by these same diagnostic measures, without increased values of HP or LP, was classified as being false negative. 

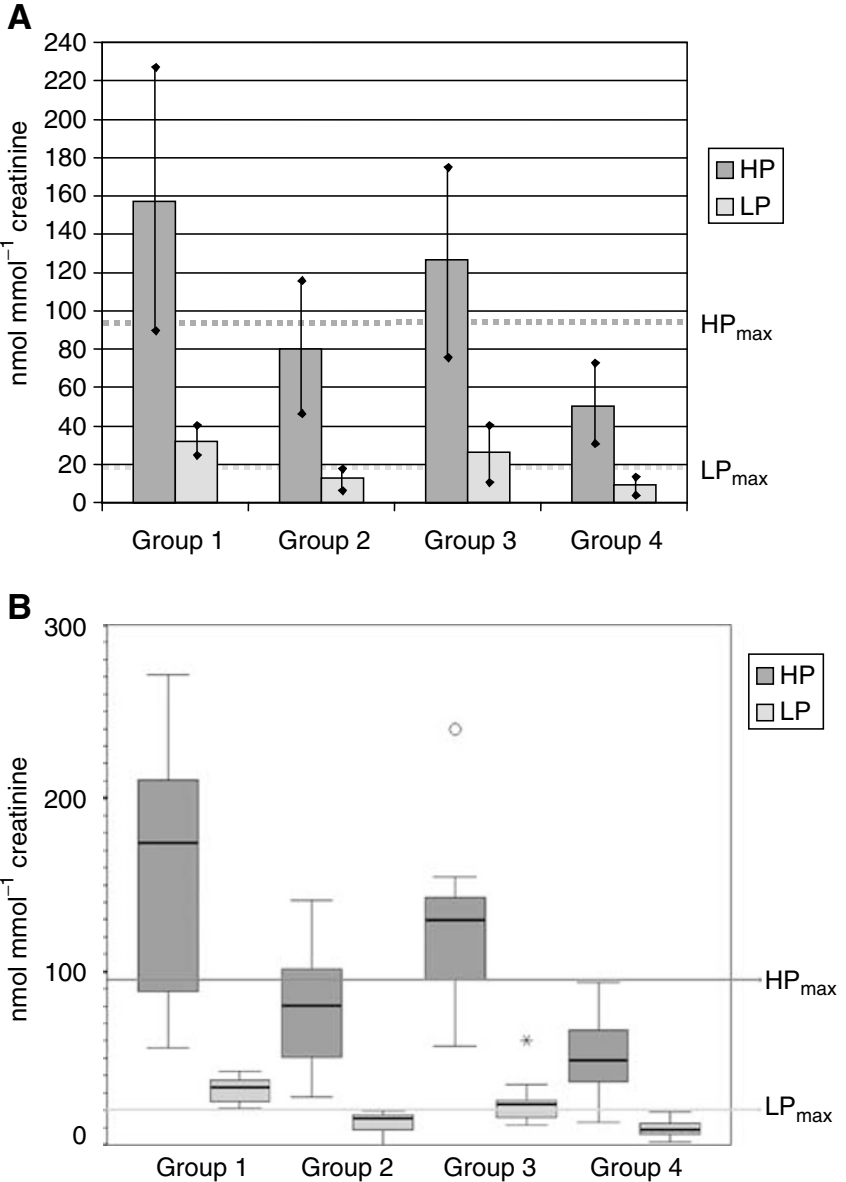

Figure I (A) Arithmetic means of concentrations of urinary HP and LP. in groups I (OSCC with mandibular bone infiltration, $n=17$ ), 2 (OSCC without mandibular bone infiltration, $n=12$ ), 3 (recurrence of OSCC, $n=13$ ) and 4 (control group, $n=74$ ). Dotted lines mark $H P_{\max }$ $\left(95 \mathrm{nmol} \mathrm{mmol}{ }^{-1}\right.$ creatinine) and $\mathrm{LP}_{\max }\left(20 \mathrm{nmol} \mathrm{mmol}^{-1}\right.$ creatinine). The urinary levels of HP in groups $1-3$ are significantly different from group 4 . The urinary concentrations of LP in groups I and 3 are significantly different from group 4. Moreover, in groups I and 3, the average urinary content of $\mathrm{HP}$ and $\mathrm{LP}$ exceeded $\mathrm{HP}_{\max }$ and $\mathrm{LP}_{\max }$ significantly. Please note that in group 2. HP is significantly elevated as a marker of increased tissue turnover as compared to group 4, but does not exceed $\mathrm{HP}_{\max }$. Length of vertical bars: standard deviation times 1.96. (B) Medians of concentrations of urinary HP and LP: Each box shows the median, quartiles and extreme values of groups I (OSCC with mandibular bone infiltration, $n=17$ ), 2 (OSCC without mandibular bone infiltration, $n=12$ ), 3 (recurrence of OSCC, $n=13$ ) and 4 (control group, $n=74$ ). Lines mark $\mathrm{HP}_{\max }$ $\left(95 \mathrm{nmol} \mathrm{mmol}{ }^{-1}\right.$ creatinine) and $\mathrm{LP}_{\max }\left(20 \mathrm{nmol} \mathrm{mmol}^{-1}\right.$ creatinine). The urinary levels of HP in groups $1-3$ are significantly different from group 4 . The urinary concentrations of LP in groups I and 3 are significantly different from group 4. Moreover, in groups $I$ and 3, the urinary content of $H P$ and $L P$ exceeded $H P_{\max }$ and $L P_{\max }$, significantly. As regards groups I and 2 , the $L P_{\max }$ line is separating LP values, completely. $O$ and $*$ label values of one patient, which significantly exceeded the normal range in the recurrence group.

Normal levels of HP and LP in the presence of a confirmed recurrence was also classified as being false negative.

In seven out of 13 patients in group 3, samples had been obtained 6 months prior to the recurrence (group 3b). For these seven patients, a paired $t$-test was performed taking both the former and the current value into account. The sensitivity and specificity in detecting the presence of OSCC tissue was evaluated. The urinary concentrations of HP and LP in the patients of groups 1-3 were compared to the urinary concentrations of HP and LP of the patients of group 4 and a receiver operator characteristic curve (ROC curve) constructed.

\section{Ethics}

The study was conducted in accordance with the standards of the Ethics Committee of the University of Kiel (chairman: Jürgen Schaub, MD, PhD, Professor for Pediatrics, Head of the Department of Pediatrics, University of Kiel, Germany; registration number of the present study: AZ D 309/01) and with the Helsinki Declaration of 1983. The patients were informed about the aim and design of the study and written consent was obtained.

\section{RESULTS}

In all, 475 urine samples from 116 patients were analysed in the course of the present study. The average urinary levels of HP and $\mathrm{LP}$ in $\mathrm{nmol} \mathrm{mmol}{ }^{-1}$ creatinine are graphically represented in Figure $1 \mathrm{~A}$ and $\mathrm{B}$. The standard deviation is shown in brackets, and the ratio of HP:LP given as follows:

Group 1: $\quad$ HP: 157.3 (68.9); LP: 31.6 (7.5); HP/LP: 4.9 (1.7);

Group 2: $\quad$ HP: 80.0 (35.4); LP: 12.9 (9.9); HP/LP: 5.5 (2.1);

Group 3: $\quad$ HP: 119.7 (47.0); LP: 23.5 (12.2); HP/LP: 5.6 (2.4);

Group 3b: HP: 59.2 (20.1); LP: 11.4 (3.4); HP/LP: $5.2(0.85)$.

Group 4: $\quad$ HP: 50.5 (20.7); LP: 9.5 (4.8); HP/LP: 6.0 (2.7).

$\mathrm{HP}_{\max }$ was $95 \mathrm{nmol} \mathrm{mmol}^{-1}$ creatinine and $\mathrm{LP}_{\max } 20 \mathrm{nmol}$ $\mathrm{mmol}^{-1}$ creatinine. Differences between the average concentrations of HP and LP of groups 1-4 were statistically significant (ANOVA: $P<0.001$, Kruskal-Wallis: $P<0.001$ ). The average urinary HP and LP concentrations were significantly elevated in group 1 compared to group $2(P<0.001$ for HP and LP), in group 1 compared to group $4(P<0.001$ for HP and LP) and in group 3 compared to group $4(P=0.001$ for HP and $P<0.01$ for LP) (Figures 1-3). The average urinary HP concentration in group 2 was significantly elevated compared to group $4(P<0.05)$. When comparing the average HP/LP ratio, it was found to be significantly lower in group 1 compared to group $4(P<0.05)$. No significant differences were found between the average HP/LP ratios of the other groups. A significant increase was found for HP $(P=0.003)$ and LP $(P=0.02)$ at the time of the recurrence when comparing group 3 with group $3 \mathrm{~b}$.

The average urinary concentrations of both HP and LP were elevated in groups 1 and $3(P<0.025)$. The average urinary content of $\mathrm{HP}$ and $\mathrm{LP}$ of group 2 was less than $\mathrm{HP}_{\max }$ and $\mathrm{LP}_{\max }$ (Figure $1 \mathrm{~A}, \mathrm{~B})$. At this level of $\mathrm{HP}_{\max }$, measurement of $\mathrm{HP}$ had a sensitivity of $61.5 \%$ and a specificity of $76.6 \%$ in separating groups 1 and 2 . $\mathrm{LP}_{\max }$ at the cutoff points chosen had a sensitivity and a specificity of $100 \%$ for separating groups 1 and 2 . Sensitivity and specificity of the present assay in regard to the detection of the presence of OSCC tissue (groups 1-3 vs group 4) was evaluated. The ROC curve showed a cutoff of $55.7 \mathrm{nmol} \mathrm{mmol}^{-1}$ creatinine for HP with a sensitivity of $90 \%$ and a specificity of $63 \%$, and a cutoff of $11.15 \mathrm{nmol} \mathrm{mmol}^{-1}$ creatinine for LP with a sensitivity of $90 \%$ and specificity of $69 \%$.

\section{DISCUSSION}

We intended to assess the ability of HP and LP as urinary markers of connective tissue and bone turnover in patients with OSCC in detecting bony invasion by malignant disease, as well as serving as a marker for tumour recurrence. We were able to show that the measurement of LP in the urine could separate groups 1 (OSCC with bone infiltration) and 2 (OSCC without bone infiltration) with a sensitivity of $100 \%$ and a specificity of $100 \%$ while no false negatives or false positives were observed. We suggest that when 

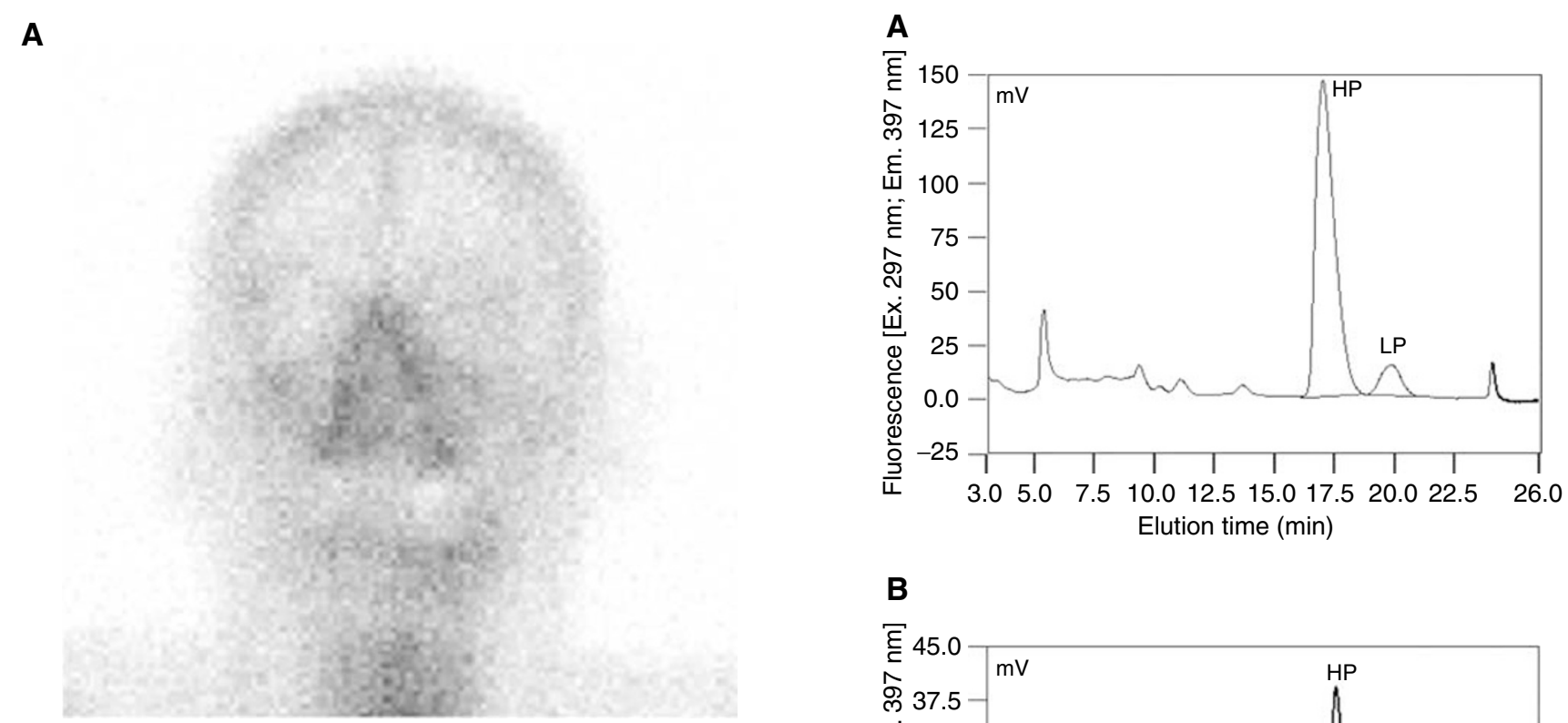

B

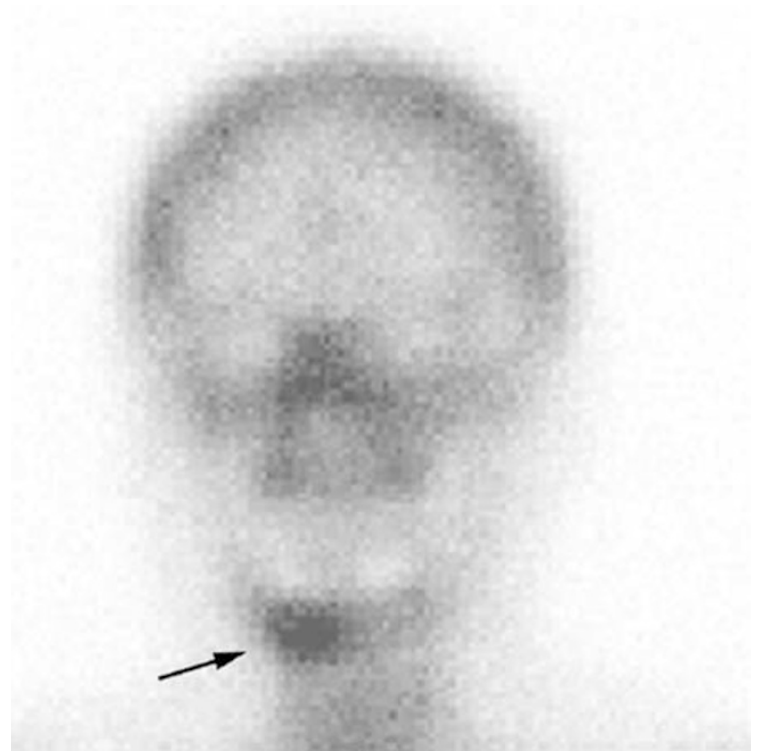

Figure 2 (A,B) Scintigraphy of a patient with an OSCC with $(\mathbf{A})$ and without (B) infiltration of the mandibular bone. Technetium $99 \mathrm{~m}$ MDP bone scans with planar imaging and SPECT were performed in the course of the staging. No increased activity is visible in $(\mathbf{A})$, but a lesion of the right mandible is seen in (B) (arrow). For the chromatograms of these patients see Figure $3 \mathrm{~A}$ and $\mathrm{B}$.

the level of LP exceeds $\mathrm{LP}_{\max }$ in a patient with a confirmed OSCC, bony invasion by the malignant process is highly likely and further investigation is required. We furthermore suggest that a urinary level of LP less than $\mathrm{LP}_{\max }$ in patients with OSCC may obviate the need for further investigations. The measurement of both LP and $\mathrm{HP}$ in the urine is recommended for the detection of bony metastases in a multitude of malignancies, indicating a higher specificity of LP for bone turnover, as compared to HP alone (Pecherstorfer et al, 1995; Takeuchi et al, 1996; Papatheofanis, 1997; Walne et al, 1997; Coleman et al, 1999; Marttunen et al, 1999; Tamura et al, 1999; Woitge et al, 1999, 2001; Demers et al, 2000; Liubimova et al, 2000; Tamada et al, 2001). Urinary HP is not specific for bony invasion, but may nevertheless be increased in
B

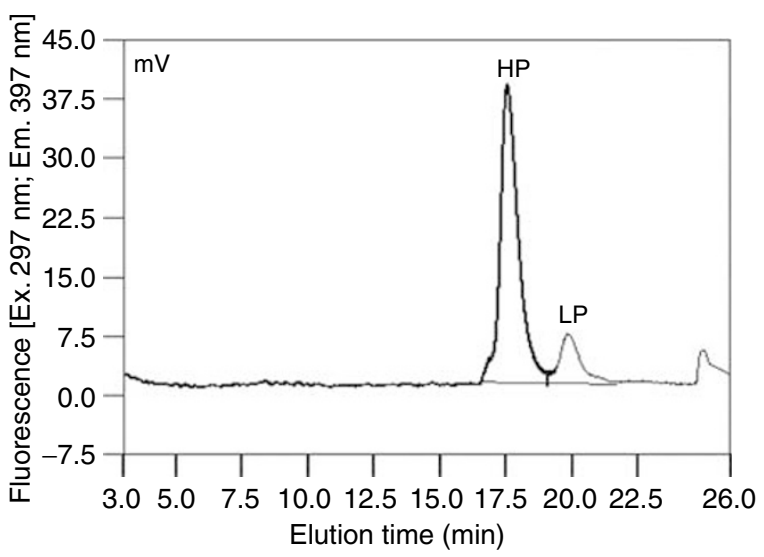

C

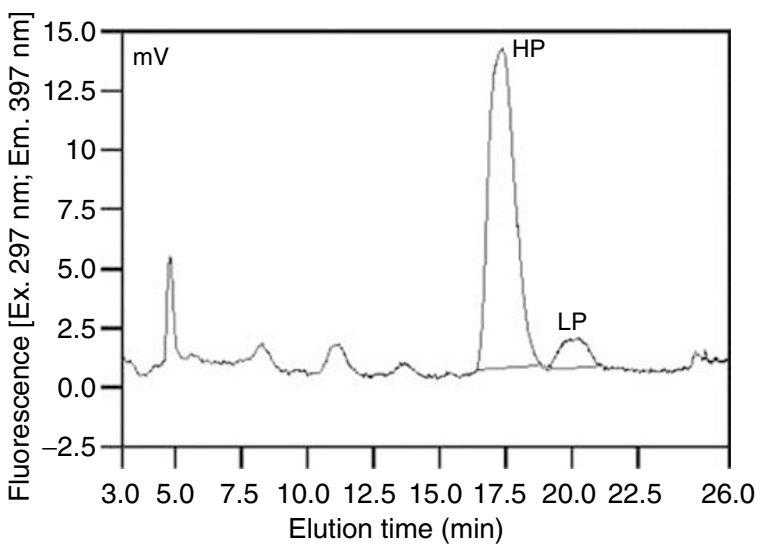

Figure 3 (A-C) Chromatograms of a patient with OSCC with $(\mathbf{A})$ and without (B) infiltration of the mandibular bone and a patient with no cancer disease $(\mathbf{C})$. The fluorescence was monitored with excitation at $297 \mathrm{~nm}$ and emission at $397 \mathrm{~nm}$. The HP peak arose at $17.5 \mathrm{~min}$ after injection, followed by the LP peak. Please note the increased HP and LP peaks in (B) compared to $(\mathbf{A})$. Please note the low HP and LP levels of the patient without current evidence of tumour $(\mathbf{C})$. For the scintigraphies of patients $(\mathbf{A})$ and $(\mathbf{B})$ see Figure $2 \mathrm{~A}$ and B. Please note that the peaks appear to be of the same size, but that the scale is different.

the urine of patients with bony metastases as compared to patients without bony metastases. Increased HP values in patients with bony invasion may be related to the presence of tumour tissue alone. This assumption is based on the fact that the average 
urinary HP concentration was significantly higher in patients with OSCC without mandibular bone invasion (group 2) as compared to the control group. Urinary LP was not significantly increased in patients of group 2 as compared to patients of group 4 further indicating specificity for bone turnover. It has been shown that neonatal rat aortic smooth muscle cells produce detectable amounts of HP (Stone et al, 1992), indicating that HP is synthesised in blood vessel walls.

The average urinary concentration of HP in group 2 did not exceed $\mathrm{HP}_{\max }$, although the arithmetic mean of the urinary concentration of HP in group 2 is significantly different from the average urinary concentration of $\mathrm{HP}$ in the control group. Therefore, the urinary levels of HP were not able to separate group 2 from group 4 . The urinary $\mathrm{HP}$ may be below $\mathrm{HP}_{\max }$ in a patient with an active malignant process (see below). We nonetheless suggest that the measurement of HP in the urine may be helpful in screening for tumour recurrence.

Groups 3 and 4 could be separated by the measurement of urinary $\mathrm{HP}$ and LP, since average urinary concentrations exceeded $\mathrm{HP}_{\max }$ and $\mathrm{LP}_{\max }$. The differences in the arithmetic means of $\mathrm{HP}$ and LP of group 3 as compared to group 4 were highly significant. We suggest that cancer in the patient history does not preclude that patient from being used as a control (group 4) if the tumour was smaller than T2 in size, was successfully treated and showed no signs of recurrence. If we had compared group 3 with a control group with no cancer in the patient history, the difference might have been even greater than observed in this study. However, we suggest that this may not be the case in the present study and that group 4 (control group) may have served as a reliable control group in this paper leading to $\mathrm{HP}_{\max }$ and $\mathrm{LP}_{\max }$ values that could separate groups 1 (bone infiltration) and 2 (no bone infiltration) with a sensitivity and a specificity of $100 \%$.

In this study, the increased turnover of collagen was indicative of tumour progression. Calculation of the ROC curve revealed a sensitivity of $90 \%$ for the presence of tumour progression if the cutoff is chosen at $55.7 \mathrm{nmol} \mathrm{mmol}{ }^{-1}$ creatinine for HP and at

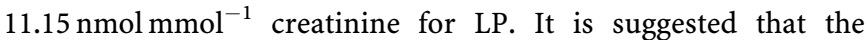
measurement of LP and HP in the urine of patients after treatment of cancer may be used in the course of recall examinations with the ultimate goal of increasing the chances of early detection of a recurrence. Patients in groups 3 and $3 \mathrm{~b}$ indicate that periodic monitoring of the collagen crosslink excretion may be useful in establishing individual trends in relation to the baseline value of HP and LP excretion. Increased values during the first 6 months following surgery may not indicate relapse if the trend is stable or decreasing. Normal values may indicate a recurrence if the levels increase. If the collagen crosslink excretion increases in recall patients, recurrence or metastasis is strongly suggested, unless other reasons for an increase in tissue turnover are found.

In the course of this part of the study, we were looking at group 3 with no special interest as to whether bone was infiltrated by the disease or not. In fact, in a large fraction of these patients bone infiltration was present. However, we were interested in HP and LP as an aid in detecting patients in cancer recall hours with increased tissue resorption.

\section{REFERENCES}

Acil Y, Brinckmann J, Notbohm H, Muller PK, Batge B (1996) Changes with age in the urinary excretion of hydroxylysylpyridinoline (HP) and lysylpyridinoline (LP). Scand J Clin Lab Invest 56: 275-283

Acil Y, Müller PK (1994) A rapid method for the isolation of the mature collagen cross-links, hydroxylysylpyridinoline (HP) and lysylpyridinoline (LP). J Chromatogr A 664: 183-188

Acil Y, Terheyden H, Dunsche A, Fleiner B, Jepsen S (2000) Threedimensional cultivation of human osteoblast-like cells on highly porous natural bone mineral. J Biomed Mater Res 51: 703-710
In all, 105 further patients, in whom urinary levels were obtained, had to be excluded from the study on the basis of the exclusion criteria stated above. Of these 105 excluded patients, 33 (31.4\%) had elevated concentrations of HP and LP beyond our suggested levels of $\mathrm{HP}_{\max }$ and $\mathrm{LP}_{\max }$. In total, 17.6\% $(n=39$ / $(105+116))$ of the recall patients were false positive, meaning that their $\mathrm{HP}$ and $\mathrm{LP}$ were greater than $\mathrm{Hp}_{\max }$ and $\mathrm{LP}_{\max }$ but there was no evidence to confirm the presence of a recurrence. We suggest that this should nonetheless at least alert the clinician to the possibility of a recurrence. Of these false-positive samples, $66.7 \%$ $(n=26 / 39)$ may have been because of a surgical procedure performed less than 6 months prior to obtaining the sample of urine, whereas $33.3 \%$ of the false-positive samples $(n=13 / 39)$ were because of the presence of active sites of inflammation. Seven of these 13 patients had confirmed osteomyelitis of the mandibular bone without evidence of tumour recurrence. No false-negative results were obtained, meaning that there were no patients with normal values where a recurrence was subsequently documented. The follow-up period has been greater than 6 months for all recall patients.

The $17.6 \%$ false-positive rate in the recall patients excluded from the study was because of surgical procedures having been performed in the 6 months prior to the investigation, or inflammatory disease such as osteomyelitis. Other possible causes for false positives are trauma, further malignancy or bone disease such as osteoporosis, rheumatoid arthritis, primary hyperparathyroidism, active Paget's disease of the bone, Ehlers-Danlos syndrome type VI and others (Eyre, 1992; Miyamoto et al, 1994; Acil et al, 1995; Chen et al, 1996; Coleman et al, 1999; Woitge et al, 1999). Urinary HP and LP are valuable trend markers of diseases with altered bone resorption as well as their treatment (Eyre, 1992; Acil et al, 1996; Coleman et al, 1997; Liubimova et al, 2000). Osteomyelitis may be added to this list following this study, since it led to a significant amount of false positives in the group of our recall patients (seven out of 39 false positives). The study has been continued monitoring the course and treatment response of mandibular osteomyelitis.

The cost of detection of urinary HP and LP is low when performed in a clinical laboratory on a routine basis. Efforts are continually being sought to detect bony invasion by OSCC (Minn et al, 1993; Huntley et al, 1996; Baker et al, 2001; Hong et al, 2001; Schimmele, 2001; Schimming et al, 2001). Clinical examination, ultrasonography and the analysis of a urine sample may provide sufficient information for treatment planning, reducing the exposure of the patient to radiation.

\section{ACKNOWLEDGEMENTS}

We gratefully acknowledge our laboratory technicians Messrs Gisela Otto and Mojgan Paymard for their assistance with the analytical procedures. The Department of Oral and Maxillofacial Surgery, University of Kiel, Germany, financed this project. We would like to express our gratitude towards Professor F Härle, $\mathrm{PhD}$, Head of the Department, for his support.
Acil Y, Vetter U, Brenner R, Muller PK, Brinckmann J (1995) Ehlers-Danlos syndrome type VI: cross-link pattern in tissue and urine sample as a diagnostic marker. J Am Acad Dermatol 33: 522-524

Baker A, McMahon J, Parmar S (2001) Immediate reconstruction of continuity defects of the mandible after tumor surgery. J Oral Maxillofac Surg 59: $1333-1339$

Body JJ, Delmas PD (1992) Urinary pyridinium cross-links as markers of bone resorption in tumor-associated hypercalcemia. J Clin Endocrinol Metab 74: 471-475 
Chen JT, Hosoda K, Hasumi K, Ogata E, Shiraki M (1996) Serum Nterminal osteocalcin is a good indicator for estimating responders to hormone replacement therapy in postmenopausal women. J Bone Miner Res 11: $1784-1792$

Coleman RE, Purohit OP, Black C, Vinholes JJ, Schlosser K, Huss H, Quinn KJ, Kanis J (1999) Double-blind, randomised, placebo-controlled, dosefinding study of oral ibandronate in patients with metastatic bone disease. Ann Oncol 10: 311-316

Coleman RE, Purohit OP, Vinholes JJ, Zekri J (1997) High dose pamidronate: clinical and biochemical effects in metastatic bone disease. Cancer 80: $1686-1690$

Demers LM, Costa L, Lipton A (2000) Biochemical markers and skeletal metastases. Cancer 88: 2919-2926

Eyre D (1992) New biomarkers of bone resorption. J Clin Endocrinol Metab 74: $470 \mathrm{~A}-470 \mathrm{C}$

Fontana A, Delmas PD (2000) Markers of bone turnover in bone metastases. Cancer 88: $2952-2960$

Hong SX, Cha IH, Lee EW, Kim J (2001) Mandibular invasion of lower gingival carcinoma in the molar region: its clinical implications on the surgical management. Int J Oral Maxillofac Surg 30: 130-138

Huntley TA, Busmanis I, Desmond P, Wiesenfeld D (1996) Mandibular invasion by squamous cell carcinoma: a computed tomographic and histological study. Br J Oral Maxillofac Surg 34: 69-74

Izumi M, Nakanishi Y, Takayama K, Kimotsuki K, Inoue K, Wataya $H$, Minami T, Hara N (2001) Diagnostic value of bone-turnover metabolites in the diagnosis of bone metastases in patients with lung carcinoma. Cancer 91: $1487-1493$

Liubimova NV, Bronnikov II, Robins SP, Trapeznikova MF, Kushlinskii NE (2000) The biochemical markers of bone remodeling in cancer patients with skeletal involvement. Vopr Onkol 46: 290-297

Marttunen MB, Hietanen P, Titinen A, Roth HJ, Viinikka L, Ylikorkala O (1999) Effects of tamoxifen and toremifene on urinary excretion of pyridinoline and deoxypyridinoline and bone density in postmenopausal patients with breast cancer. Calcif Tissue Int 65: 365-368

Minn H, Aitasalo K, Happonen RP (1993) Detection of cancer recurrence in irradiated mandible using positron emission tomography. Eur Arch Otorhinolaryngol 250: 312 - 315

Miyamoto KK, McSherry SA, Robins SP, Besterman JM, Mohler JL (1994) Collagen cross-link metabolites in urine as markers of bone metastases in prostatic carcinoma. J Urol 151: 909-913

Papatheofanis FJ (1997) Quantitation of biochemical markers of bone resorption following strontium-89-chloride therapy for metastatic prostatic carcinoma. J Nucl Med 38: 1175-1179

Pecherstorfer M, Zimmer-Roth I, Schilling T, Woitge HW, Schmidt H, Baumgartner G, Thiebaud D, Ludwig H, Seibel MJ (1995) The diagnostic value of urinary pyridinium cross-links of collagen, serum total alkaline phosphatase, and urinary calcium excretion in neoplastic bone disease. $J$ Clin Endocrinol Metab 80: $97-103$

Reichart PA, Philipsen HP (1999) Maligne tumoren. In Oralpathologie, Rateitschak KH, Wolf HF (eds) pp 108-118. Farbatlanten der Zahnmedizin. Thieme: Stuttgart

Schimmele SR (2001) Delayed reconstruction of continuity defects of the mandible after tumor surgery. J Oral Maxillofac Surg 59: $1340-$ 1344

Schimming R, Juengling FD, Altehofer C, Schmelzeisen R (2001) Diagnosis of questionable mandibular infiltration by squamous epithelial carcinomas. 3-D 99mTc-DPD SPECT reconstruction and (18F) fluoride PET study: diagnostic advantages or unnecessary expense? HNO 49: $355-360$

Stone PJ, Bryan-Rhadfi J, Shaw HA, Franzblau C (1992) Isolation of hydroxylysyl pyridinoline, a mature collagen crosslink from neonatal rat aorta smooth muscle cell cultures. Matrix 12: $381-387$

Takeuchi S, Arai K, Saitoh H, Yoshida K, Miura M (1996) Urinary pyridinoline and deoxypyridinoline as potential markers of bone metastasis in patients with prostate cancer. J Urol 156: $1691-1695$

Tamada T, Sone T, Tomomitsu T, Jo Y, Tanaka H, Fukunaga M (2001) Biochemical markers for the detection of bone metastasis in patients with prostate cancer: diagnostic efficacy and the effect of hormonal therapy. J Bone Miner Metab 19: $45-51$

Tamura H, Ishii S, Ikeda T, Enomoto K, Kitajima M (1999) The relationship between urinary pyridinoline, deoxypyridinoline and bone metastasis in a rat breast cancer model. Breast Cancer 6: 23-28

Vinholes J, Guo CY, Purohit OP, Eastell R, Coleman RE (1996) Metabolic effects of pamidronate in patients with metastatic bone disease. $\mathrm{Br} J$ Cancer 73: 1089 - 1095

Walne AJ, Jenkins PJ, James IT, Plowman PN (1997) Pyridinium crosslinks in the monitoring of patients with bone metastases from carcinoma of the breast. Clin Oncol 9: 30-34

Woitge HW, Pecherstorfer M, Horn E, Keck AV, Diel IJ, Bayer P, Ludwig H, Ziegler R, Seibel MJ (2001) Serum bone sialoprotein as a marker of tumour burden and neoplastic bone involvement and as a prognostic factor in multiple myeloma. Br J Cancer 84: 344-351

Woitge HW, Pecherstorfer M, Li Y, Keck AV, Horn E, Ziegler R, Seibel MJ (1999) Novel serum markers of bone resorption: clinical assessment and comparison with established urinary indices. J Bone Miner Res 14: 792 801

Yoshida K, Sumi S, Arai K, Koga F, Umeda H, Hosoya Y, Honda M, Yano M, Moriguchi H, Kitahara S (1997) Serum concentration of type I collagen metabolites as a quantitative marker of bone metastases in patients with prostate carcinoma. Cancer 80: 1760-1767 